\title{
An Increased Ratio of Glycated Albumin to HbA1c Is Associated with the Degree of Liver Fibrosis in Hepatitis B Virus-Positive Patients
}

\author{
Hirayuki Enomoto, ${ }^{1}$ Nobuhiro Aizawa, ${ }_{1}^{1}$ Hideji Nakamura, ${ }^{2}$ Yoshiyuki Sakai, \\ Yoshinori Iwata, ${ }^{1}$ Hironori Tanaka, ${ }^{1}$ Naoto Ikeda, ${ }^{1}$ Tomoko Aoki, ${ }^{1}$ Yukihisa Yuri, ${ }^{1}$ \\ Kazunori Yoh, ${ }^{1}$ Kenji Hashimoto, ${ }^{1}$ Akio Ishii, ${ }^{1}$ Tomoyuki Takashima, ${ }^{1}$ Kazunari Iwata, \\ Masaki Saito, ${ }^{1}$ Hiroyasu Imanishi, ${ }^{1}$ Hiroko Iijima, ${ }^{1}$ and Shuhei Nishiguchi ${ }^{1}$ \\ ${ }^{1}$ Division of Hepatobiliary and Pancreatic Disease, Department of Internal Medicine, Hyogo College of Medicine, Mukogawa-cho 1-1, \\ Nishinomiya, Hyogo 663-8501, Japan \\ ${ }^{2}$ Department of Gastroenterology and Hepatology, Nissay Hospital, Itachibori 6-3-8, Nishi-ku, Osaka 550-0012, Japan \\ Correspondence should be addressed to Hirayuki Enomoto; enomoto@hyo-med.ac.jp
}

Received 29 November 2013; Revised 7 January 2014; Accepted 7 January 2014; Published 17 February 2014

Academic Editor: Edoardo Giovanni Giannini

Copyright (C) 2014 Hirayuki Enomoto et al. This is an open access article distributed under the Creative Commons Attribution License, which permits unrestricted use, distribution, and reproduction in any medium, provided the original work is properly cited.

Background. In hepatitis B virus- (HBV-) positive patients, the relationship between the metabolic variables and histological degree of liver fibrosis has been poorly investigated. Methods. A total of $176 \mathrm{HBV}$-positive patients were assessed in whom the ratios of glycated albumin-to-glycated hemoglobin (GA/HbAlc) were calculated in order to investigate the relationship with the degree of liver fibrosis. Results. The GA/HbAlc ratio increased in association with the severity of fibrosis (METAVIR scores: F0-1: 2.61 \pm $0.24, F 2: 2.65 \pm 0.24, F 3: 2.74 \pm 0.38$, and F4: $2.91 \pm 0.63)$. The GA/HbAlc ratios were inversely correlated with four variables of liver function: the prothrombin time (PT) percentage $(P<0.0001)$, platelet count $(P<0.0001)$, albumin value $(P<0.0001)$, and cholinesterase value $(P<0.0001)$. The GA/HbAlc ratio was positively correlated with two well-known markers of liver fibrosis, FIB$4(P<0.0001)$ and the AST-to-platelet ratio index (APRI) $(P<0.0001)$. Furthermore, the GA/HbAlc showed better correlations with two variables of liver function (PT percentage and cholinesterase value) than did FIB-4 and with all four variables than did the APRI. Conclusion. The GA/HbAlc ratio is associated with the degree of liver fibrosis in HBV-positive patients.

\section{Introduction}

In patients with chronic liver disease (CLD), liver biopsy is the gold standard method to evaluate the degree of liver fibrosis [1]. However, a liver biopsy is a costly and invasive technique associated with a risk of complications. In addition, there can be sampling errors, because only $1 / 50,000$ of the organ is used for the analysis [1]. Furthermore, it has been reported that there are inter- and intraobserver discrepancies of $10 \%$ to $20 \%$ for biopsy samples $[2,3]$. Therefore, many noninvasive markers of fibrosis available via laboratory tests have been reported, and hepatitis $\mathrm{C}$ virus- (HCV-) positive patients have provided a good research base in this context.
It is known that significant differences are observed between $\mathrm{HCV}$-positive patients and hepatitis B virus- (HBV-) positive patients, not only in the etiology, but also in terms of many other clinical parameters, including the natural history of the disease, the laboratory parameters, and the liver histology $[4,5]$. However, the number of reports regarding fibrosis markers for HBV-positive patients is much lower than that for HCV-positive patients. In particular, there have been few reports about the relationship between the metabolic parameters and histological degree of liver fibrosis in HBVpositive patients, despite the fact that the liver functions as an important metabolic organ. 
The values of glycated proteins reflect the plasma glucose level, and glycated hemoglobin (HbAlc) is commonly used as a reliable index of glycemic control in diabetic patients $[6,7]$. The turnover period of hemoglobin in erythrocytes is about four months, and the HbAlc level therefore reflects the plasma glucose levels for the past few months [8]. Glycated albumin (GA) is another marker of the glycemic control during the past few weeks, because the turnover of albumin is about three weeks $[9,10]$. In patients with CLD, hypersplenism abbreviates the lifespan of erythrocytes, leading to lower $\mathrm{HbAlc}$ values relative to the degree of glycemia. In contrast, the GA levels in CLD patients are higher than those estimated based on the levels of glycemia, because the turnover of serum albumin in CLD patients is increased as a result of the compensation for the decreased albumin production in the liver [11]. Since the HbAlc shows lower values and the GA shows higher values in CLD patients, the GA/HbAlc ratio is predicted to be high in patients with $\mathrm{CLD}$. Indeed, the GA/HbAlc ratio has been reported to be associated with the histological stage of liver fibrosis and portal hypertension in HCV-positive CLD and nonalcoholic steatohepatitis [12-15]. In the present study, we investigated the GA/HbAlc ratio in $\mathrm{HBV}$-positive patients and its correlation with liver fibrosis.

\section{Materials and Methods}

2.1. Patients. We studied a total of $173 \mathrm{HBV}$-positive patients who had undergone percutaneous liver biopsies between January 2008 and March 2010 at our institution. This study was retrospective and consecutively included all patients who fulfilled the following conditions: (1) HBV infection diagnosed by positive HBsAg status for at least six months. (2) Blood samples, including samples for an analysis of the GA and HbAlc levels, were obtained on the same day as the liver biopsies. Patients with the following conditions were excluded from the study: the presence of other liver diseases, hepatocellular carcinoma, immunosuppressive therapy, $\mathrm{HCV}$ coinfection, and insufficient liver tissue for the staging of fibrosis (a minimum of $15 \mathrm{~mm}$ of liver tissue with five or more portal tracts was required for diagnosis). The present study did not include patients whose GA/HbAlc ratios could have been influenced by poorly controlled diabetes.

The characteristics of the study population are summarized in Table 1 . The study conformed to the ethical guidelines of the Declaration of Helsinki, and written informed consent regarding the liver biopsy and use of clinical data was obtained from all patients on admission. This study was approved by the ethics committee of the institutional review board.

2.2. Laboratory Data and Liver Biopsy. The HbAlc was measured by high-performance liquid chromatography, with calibration using Japan Diabetes Society (JDS) Lot $2[15,16]$. The value for HbAlc (\%) was estimated as a NGSP equivalent value (\%) calculated using the following formula: in the range of JDS values $\leq 4.9 \%$ : NGSP $(\%)=$ JDS $(\%)+0.3 \%$ and in the range of JDS 5.0-9.9\%: NGSP (\%) = JDS (\%) +
TABLE 1: The characteristics of the 173 hepatitis B virus- (HBV-) positive patients.

\begin{tabular}{lc}
\hline Age (years) & $46(25-79)$ \\
Gender (male/female) & $96 / 77$ \\
AST (IU/L) & $27(11-269)$ \\
ALT (IU/L) & $28(7-680)$ \\
$\gamma$-GTP (IU/L) & $25(7-349)$ \\
ALP (IU/L) & $203(71-835)$ \\
Total bilirubin (mg/dL) & $0.8(0.1-2.3)$ \\
Albumin (g/dL) & $3.90 \pm 0.40$ \\
Hemoglobin (g/dL) & $13.5 \pm 3.8$ \\
Platelets (×10 $\left.{ }^{3} / \mu \mathrm{L}\right)$ & $178 \pm 72$ \\
PT (\%) & $89.8 \pm 12.3$ \\
Diabetes mellitus (present/absent) & $6 / 167$ \\
Glucose (mg/dL) & $91.3 \pm 13.9$ \\
Triglyceride (mg/dL) & $99.0 \pm 45.5$ \\
Total cholesterol (mg/dL) & $177 \pm 32$ \\
Body mass index & $22.9 \pm 4.1$ \\
HBV-DNA (log copies/mL) & 3.7 (n.d-over 9.1) \\
HBe antigen (positive/negative) & $59 / 114$ \\
Treatment with NAs (present/absent) & $67 / 106$ \\
Histological stage of liver fibrosis (F0-1/F2/F3/F4) & $94 / 38 / 28 / 13$ \\
\hline n.d: not detectable; NAs: nucleoside/nucleotide analogues. \\
${ }^{*}$ HBV-DNA ranged from undetectable level in patients \\
of NAs to over measurable level (9.1 log copies/mL) in patients without \\
treatment. & \\
& \\
&
\end{tabular}

$0.4 \%$ [17]. Routine laboratory studies, including platelet counts, the prothrombin time (PT) percentage, and liver function tests (ALT, AST, alkaline phosphatase, albumin, and cholinesterase), were also performed.

In the present study, the values of two biomarkers associated with the progression of liver fibrosis (FIB-4 and the APRI, the AST-to-platelet count ratio index) were calculated, because these markers were previously shown to be associated with the progression of liver fibrosis [18-20]. The FIB-4 and APRI values were calculated based on formulas developed by Vallet-Pichard et al. [21] and Wai et al. [22], respectively: FIB- $4=$ Age [years] $\times$ AST $[\mathrm{U} / \mathrm{L}] /($ platelets $\left.\left[10^{9} / \mathrm{L}\right] \times(\mathrm{ALT}[\mathrm{U} / \mathrm{L}])^{1 / 2}\right)$, in which the age of the patient is the age at the time of liver biopsy and APRI $=100 \times($ AST level/upper limit of normal)/platelets $\left[10^{9} / \mathrm{L}\right]$.

Liver biopsy examinations were carried out according to the standard techniques. All liver samples were evaluated by well-trained pathologists at our institute, with an evaluation of the fibrosis stage and activity grade. Fibrosis was staged on a scale of F0-F4 (F0, no fibrosis; F1, portal fibrosis without septa; F2, portal fibrosis with rare septa; F3, numerous septa without cirrhosis; F4, liver cirrhosis) according to the METAVIR scoring system [23]. The histological findings of the biopsy tissues were also routinely evaluated in our department. All authors participated in the conferences about the histological findings, and the final results were confirmed by two authors (H. Enomoto and H. Imanishi) who received training for histological studies. 


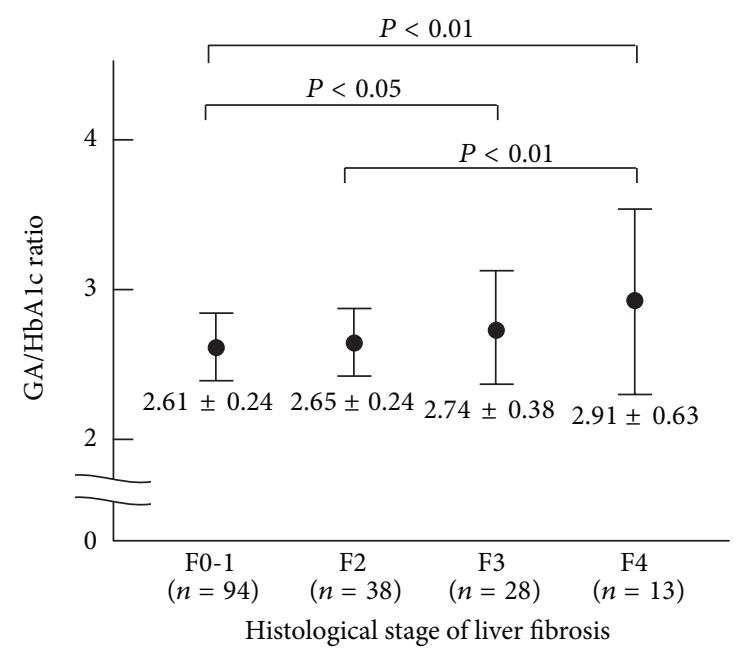

FIGURE 1: GA/HbAlc ratios in relation to the METAVIR fibrosis scores among the HBV-positive patients. The GA/HbAlc ratio increased in association with the stage of liver fibrosis. There were significant differences between the F0-F1 versus F3, F0-F1 versus F4, and $F 2$ versus $F 4$ groups.

2.3. Statistical Analysis. In the present study, we investigated whether the GA/HbAlc ratio is associated with the degree of liver fibrosis in HBV-positive patients. The data for the comparisons among the groups "F0-1 versus F2 versus F3 versus F4" was analyzed by a nonrepeated measurements ANOVA, and statistical significance was consequently evaluated with the Bonferroni correction. The relationships between the $\mathrm{GA} / \mathrm{HbAlc}$ ratio and other variables, including the FIB-4 and APRI, were evaluated with Spearman's correlation coefficient. A value of $P<0.05$ was considered to be significant.

\section{Results}

3.1. The GA/HbAlc Ratio Increases with the Histological Stage of HBV-Positive Patients. A total of $173 \mathrm{HBV}$-positive patients were included in this study. The characteristics of the study population are summarized in Table 1 . The population consisted of 96 (55.5\%) male patients and 77 (44.5\%) female patients, and the age of patients ranged from 25 to 79 years old (median 46 years old). As shown in Figure 1, the mean value of the GA/HbAlc ratio increased in association with the histological stage of liver fibrosis in the HBV-positive patients.

3.2. The GA/HbAlc Ratio Is Associated with the Laboratory Parameters in $\mathrm{HBV}$-Positive Patients. We next investigated whether the GA/HbAlc ratio was related to the laboratory parameters of the liver function, including the PT (\%), platelet count, albumin level, and cholinesterase level. Table 2 shows that there is a significant reciprocal correlation of the GA/HbAlc ratio with the PT $(\%)(R=-0.396, P<0.0001)$ and platelet count $(R=-0.421, P<0.0001)$ in HBV-positive patients. The GA/HbAlc ratio was also inversely correlated with the serum albumin level $(R=-0.332, P<0.0001)$ and the cholinesterase level $(R=-0.411, P<0.0001)$.
TABLE 2: The correlations of the three biomarkers with liver function parameters.

\begin{tabular}{lccc}
\hline & \multicolumn{3}{c}{ Correlation coefficient } \\
& FIB-4 & APRI & GA/HbAlc \\
\hline Prothrombin time (\%) & -0.362 & -0.284 & -0.396 \\
Platelet count & -0.532 & -0.372 & -0.421 \\
Albumin value & -0.372 & -0.301 & -0.332 \\
Cholinesterase value & -0.344 & -0.315 & -0.411 \\
\hline
\end{tabular}

GA/HbAlc: glycated albumin- (GA-) to-glycated hemoglobin (HbAlc) ratio. APRI: AST-to-platelet ratio index.

These findings showed that the GA/HbAlc ratio increased in association with changes in the levels of markers related to liver fibrosis.

3.3. The GA/HbA1c Ratio and Fibrosis-Related Markers in $\mathrm{HBV}$-Positive Patients. Since we found that the GA/HbAlc ratio was associated with the stage of liver fibrosis in the HBVpositive patients, we therefore investigated the relationships of the GA/HbAlc ratio with two previously established fibrosis-related markers, FIB-4 and APRI. As shown in Figure 2, the GA/HbAlc ratio was significantly correlated with the FIB-4 $(R=0.598, P<0.0001)$ and APRI $(R=0.505$, $P<0.0001)$. We examined the correlations of these biomarkers with four parameters of liver function (PT percentage, albumin value, platelet count, and cholinesterase value) and found that the GA/HbAlc ratio showed better correlations than the FIB-4 value for two parameters (PT percentage and cholinesterase value). In addition, the correlations of the GA/HbAlc ratio with the findings of liver function tests were higher than those of the APRI for all four parameters (Table 2).

\section{Discussion}

Liver biopsy is the gold standard method for histologically assessing liver fibrosis. However, a liver biopsy is an invasive procedure carrying a small risk of severe complications. In addition to the FIB-4 and APRI, noninvasive biomarkers, such as the FibroTest score [24], Forns score [25], Hepascore [26], FibroMeter [27], FibroIndex [28], and Lok index [29], were previously reported to be associated with the liver fibrosis. In the present study, we showed that the GA/HbAlc ratio is associated with the histological stage of liver fibrosis in $\mathrm{HBV}$-positive patients (Figure 1). We also showed that the $\mathrm{GA} / \mathrm{HbA1c}$ ratio was significantly related to the laboratory variables of liver function (Table 2). Among the previously reported biomarkers for liver fibrosis, the FIB-4 and APRI are simple and useful markers that can be measured using routinely available clinical parameters without any specialized equipment. We found that the GA/HbAlc ratio significantly correlated with these well-established markers in $\mathrm{HBV}$-positive patients (Figure 2). These findings suggest that there is a strong relationship between the GA/HbAlc ratio and the levels of fibrosis-related markers in HBV-positive patients. 


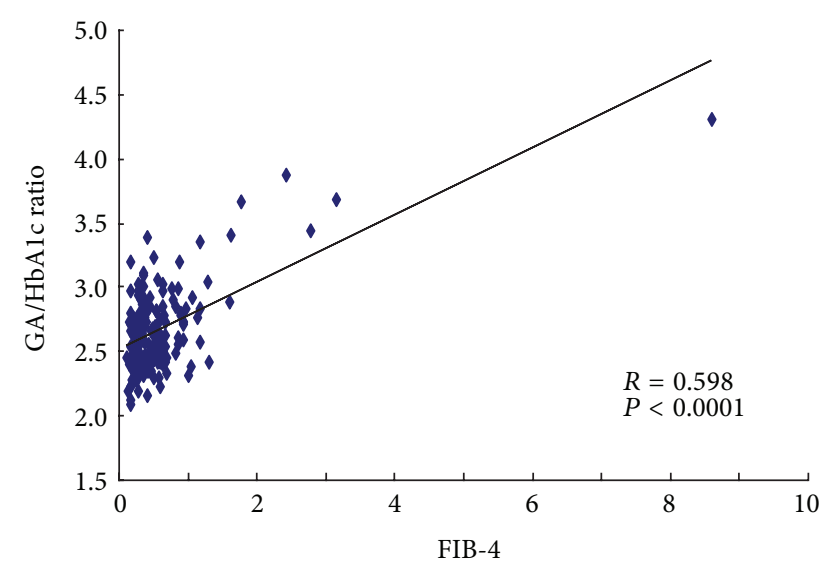

(a)

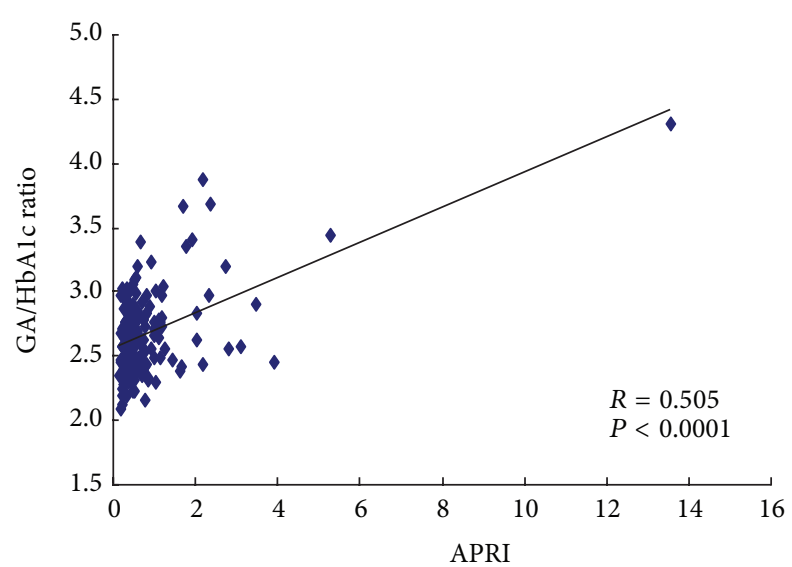

(b)

FIGURE 2: The correlation of the GA/HbAlc ratio with other fibrosis-related biomarkers. The GA/HbAlc ratio was correlated with the FIB-4 and APRI. APRI: AST-to-platelet ratio index.

When we examined the four variables of liver function, the correlation coefficients of the GA/HbAlc ratio were higher than those of the FIB-4 for two of the variables. In addition, the GA/HbAlc ratio was better correlated with all four variables examined than was the APRI (Table 2). It has been reported that the etiology of CLD influences the performance of liver fibrosis biomarkers. Unlike that observed in HCV-positive patients, noninvasive biomarkers are sometimes reported to not provide a correct evaluation of the degree of liver fibrosis in HBV-positive patients [30-32]. One major reason could be that the previously established biomarkers are obtained using calculations which include the AST and/or ALT. HBV infections sometimes show an acute liver inflammatory phase, and the AST and ALT values can therefore change from a mildly elevated level to an extremely high level in the same patient depending on the time when the patient is evaluated. In the present study, we included all HBV-positive patients without setting an upper limit for the AST and ALT levels, and some patients with remarkably elevated AST and ALT levels showed very high FIB- 4 and APRI indices. The APRI is calculated using only the AST value and platelet count, while the FIB- 4 calculation includes both AST and ALT values. Therefore, the acute elevation of AST and ALT in HBV-positive patients should more severely affect the value of the APRI than the FIB-4, although the ALT value was used as the (ALT) ${ }^{1 / 2}$ for the FIB-4 calculation. The advantage of using the GA/HbAlc ration may therefore depend on the instability of AST and ALT values in HBV-positive patients, because the GA/HbAlc ration is calculated using only the values of two glycated proteins and is independent of the AST and ALT values.

Since the liver plays a central role in metabolism, the progression of liver disease should lead to changes in metabolic parameters. However, most of the established biomarkers for liver fibrosis depend on only nonmetabolic parameters, such as the values of AST, ALT, and the platelet count. Recently, some groups, including our group, reported that the GA/HbAlc ratio was associated with the degree of liver fibrosis in various types of CLD, such as HCV-related CLD and nonalcoholic steatohepatitis [12-15]. Furthermore, we have reported that the amino acid imbalance was associated with the degree of liver fibrosis and the severity of esophageal varices in HCV-positive patients, thus suggesting that metabolism-related parameters could be potential biomarkers for the severity of CLD [33].

We herein demonstrated that the GA/HbAlc ratio increased in association with the stage of liver fibrosis in HBV-positive patients; however, the differences among the fibrosis stages were relatively small (Figure 1). Therefore, the GA/HbAlc ratio alone is not an ideal biomarker to evaluate liver fibrosis, although its correlations with the liver functional tests were as good as the previously reported well-established markers, the FIB-4 and APRI (Table 2). In addition, the present study was a simple descriptive study and did not have a prospective or longitudinal design. Therefore, we cannot draw any conclusions regarding the relationships with the progression of liver fibrosis or clinical outcomes. Recently, there was a report that it was possible to predict portal hypertension using three metabolic parameters [34]. A new biomarker based on a combination of metabolic parameters that includes the $\mathrm{GA} / \mathrm{HbAlc}$ ratio would be useful for evaluating liver fibrosis in HBV-positive patients.

\section{Conclusion}

In conclusion, we herein demonstrated that the GA/HbAlc ratio increases in association with the stage of liver fibrosis and is correlated with the levels of markers related to liver fibrosis in HBV-positive patients.

\section{Abbreviations}

GA: Glycated albumin

CLD: Chronic liver disease

HCV: Hepatitis $C$ virus

HBV: Hepatitis B virus

PT: Prothrombin time. 


\section{Conflict of Interests}

The authors declare that there is no conflict of interests regarding the publication of this paper.

\section{Acknowledgment}

This study was supported by a Grant-in-Aid for Health and Labor Sciences Research from the Ministry of Health, Labour, and Welfare of Japan.

\section{References}

[1] K. A. Gebo, H. F. Herlong, M. S. Torbenson et al., "Role of liver biopsy in management of chronic hepatitis C: a systematic review," Hepatology, vol. 36, no. 5, pp. S161-S172, 2002.

[2] P. Bedossa, D. Dargère, and V. Paradis, "Sampling variability of liver fibrosis in chronic hepatitis C," Hepatology, vol. 38, no. 6, pp. 1449-1457, 2003.

[3] A. Regev, M. Berho, L. J. Jeffers et al., "Sampling error and intraobserver variation in liver biopsy in patients with chronic HCV infection," American Journal of Gastroenterology, vol. 97, no. 10, pp. 2614-2618, 2002.

[4] Y.-F. Liaw, N. Leung, J.-H. Kao et al., "Asian-Pacific consensus statement on the management of chronic hepatitis B: a 2008 update," Hepatology International, vol. 2, no. 3, pp. 263-283, 2008.

[5] European Association for the Study of the Liver, "EASL clinical practice guidelines: management of chronic hepatitis B virus infection," Journal of Hepatology, vol. 57, no. 1, pp. 167-185, 2012.

[6] R. J. Koenig, C. M. Peterson, and R. L. Jones, "Correlation of glucose regulation and hemoglobin A(Ic) in diabetes mellitus," The New England Journal of Medicine, vol. 295, no. 8, pp. 417420, 1976.

[7] H. F. Bunn, K. H. Gabbay, and P. M. Gallop, "The glycosylation of hemoglobin: relevance to diabetes mellitus," Science, vol. 200, no. 4337 , pp. 21-27, 1978.

[8] Y. Tahara and K. Shima, "Kinetics of $\mathrm{HbA}(1 \mathrm{c})$, glycated albumin, and fructosamine and analysis of their weight functions against preceding plasma glucose level," Diabetes Care, vol. 18, no. 4, pp. 440-447, 1995.

[9] R. Dolhofer and O. H. Wieland, "Glycosylation of serum albumin: elevated glycosyl-albumin in diabetic patients," FEBS Letters, vol. 103, no. 2, pp. 282-286, 1979.

[10] C. E. Guthrow, M. A. Morris, and J. F. Day, "Enhanced nonenzymatic glucosylation of human serum albumin in diabetes mellitus," Proceedings of the National Academy of Sciences of the United States of America, vol. 76, no. 9, pp. 4258-4261, 1979.

[11] M. Koga and S. Kasayama, "Clinical impact of glycated albumin as another glycemic control marker," Endocrine Journal, vol. 57, no. 9, pp. 751-762, 2010.

[12] Y. Bando, H. Kanehara, D. Toya, N. Tanaka, S. Kasayama, and M. Koga, "Association of serum glycated albumin to haemoglobin A1C ratio with hepatic function tests in patients with chronic liver disease," Annals of Clinical Biochemistry, vol. 46, part 5, pp. 368-372, 2009.

[13] N. Aizawa, H. Enomoto, H. Imanishi et al., "Elevation of the glycated albumin to glycated hemoglobin ratio during the progression of HCV-related liver fibrosis," World Journal of Hepatology, vol. 4, no. 1, pp. 11-17, 2012.
[14] Y. Sakai, H. Enomoto, N. Aizawa et al., "Relationship between elevation of glycated albumin to glycated hemoglobin ratio in patients with a high bleeding risk of esophageal varices," Hepatogastroenterology, vol. 59, no. 119, pp. 2280-2284, 2012.

[15] Y. Bando, H. Kanehara, K. Aoki et al., “The glycated albumin to $\mathrm{HbAlc}$ ratio is increasing along with fibrosis stage in nonalcoholic steatohepatitis," Annals of Clinical Biochemistry, vol. 49, part 4, pp. 387-390, 2012.

[16] M. Tominaga, H. Makino, G. Yoshino et al., "Japanese standard reference material for JDS Lot 2 haemoglobin Alc. I: comparison of Japan Diabetes Society-assigned values to those obtained by the Japanese and USA domestic standardization programmes and by the International Federation of Clinical Chemistry reference laboratories," Annals of Clinical Biochemistry, vol. 42, part 1, pp. 41-46, 2005.

[17] A. Kashiwagi, M. Kasuga, E. Araki et al., "International clinical harmonization of glycated hemoglobin in Japan: from Japan Diabetes Society to National Glycohemoglobin Standardization Program values," Diabetology International, vol. 3, no. 1, pp. 810, 2012.

[18] V. Mallet, V. Dhalluin-Venier, C. Roussin et al., "The accuracy of the FIB-4 index for the diagnosis of mild fibrosis in chronic hepatitis B," Alimentary Pharmacology \& Therapeutics, vol. 29, no. 4, pp. 409-415, 2009.

[19] D. M. Lebensztejn, E. Skiba, M. Sobaniec-Lotowska, and M. Kaczmarski, "A simple noninvasive index (APRI) predicts advanced liver fibrosis in children with chronic hepatitis B," Hepatology, vol. 41, no. 6, pp. 1434-1435, 2005.

[20] H. Wang, L. Xue, R. Yan et al., "Comparison of FIB-4 and APRI in Chinese HBV-infected patients with persistently normal ALT and mildly elevated ALT," Journal of Viral Hepatitis, vol. 20, no. 4, pp. 3-10, 2013.

[21] A. Vallet-Pichard, V. Mallet, B. Nalpas et al., "FIB-4: an inexpensive and accurate marker of fibrosis in HCV infection. Comparison with liver biopsy and FibroTest," Hepatology, vol. 46, no. 1, pp. 32-36, 2007.

[22] C.-T. Wai, J. K. Greenson, R. J. Fontana et al., "A simple noninvasive index can predict both significant fibrosis and cirrhosis in patients with chronic hepatitis C, Hepatology, vol. 38, no. 2, pp. 518-526, 2003.

[23] The French METAVIR Cooperative Study Group, "Intraobserver and interobserver variations in liver biopsy interpretation in patients with chronic hepatitis C," Hepatology, vol. 20, no. 1, part 1, pp. 15-20, 1994.

[24] F. Imbert-Bismut, V. Ratziu, L. Pieroni, F. Charlotte, Y. Benhamou, and T. Poynard, "Biochemical markers of liver fibrosis in patients with hepatitis $C$ virus infection: a prospective study," The Lancet, vol. 357, no. 9262, pp. 1069-1075, 2001.

[25] X. Forns, S. Ampurdanès, J. M. Llovet et al., "Identification of chronic hepatitis $\mathrm{C}$ patients without hepatic fibrosis by a simple predictive model," Hepatology, vol. 36, no. 4, part 1, pp. 986-992, 2002.

[26] L. A. Adams, M. Bulsara, E. Rossi et al., "Hepascore: an accurate validated predictor of liver fibrosis in chronic hepatitis C infection," Clinical Chemistry, vol. 51, no. 10, pp. 1867-1873, 2005.

[27] P. Calès, F. Oberti, S. Michalak et al., "A novel panel of blood markers to assess the degree of liver fibrosis," Hepatology, vol. 42, no. 6, pp. 1373-1381, 2005.

[28] M. Koda, Y. Matunaga, M. Kawakami, Y. Kishimoto, T. Suou, and Y. Murawaki, "Fibrolndex, a practical index for predicting 
significant fibrosis in patients with chronic hepatitis C," Нераtology, vol. 45, no. 2, pp. 297-306, 2007.

[29] A. S. F. Lok, M. G. Ghany, Z. D. Goodman et al., "Predicting cirrhosis in patients with hepatitis $\mathrm{C}$ based on standard laboratoiy tests: results of the HALT-C cohort," Hepatology, vol. 42, no. 2, pp. 282-292, 2005.

[30] G. Sebastiani, L. Castera, P. Halfon et al., "The impact of liver disease aetiology and the stages of hepatic fibrosis on the performance of non-invasive fibrosis biomarkers: an international study of 2411 cases," Alimentary Pharmacology \& Therapeutics, vol. 34, no. 10, pp. 1202-1216, 2011.

[31] C.-T. Wai, C. L. Cheng, A. Wee et al., "Non-invasive models for predicting histology in patients with chronic hepatitis B," Liver International, vol. 26, no. 6, pp. 666-672, 2006.

[32] W. Jin, Z. Lin, Y. Xin, X. Jiang, Q. Dong, and S. Xuan, "Diagnostic accuracy of the aspartate aminotransferase-to-platelet ratio index for the prediction of hepatitis B-related fibrosis: a leading meta-analysis," BMC Gastroenterology, vol. 12, article 14, 2012.

[33] H. Enomoto, Y. Sakai, N. Aizawa et al., "Association of amino acid imbalance with the severity of liver fibrosis and esophageal varice," Annals of Hepatology, vol. 12, no. 3, pp. 471-478, 2013.

[34] M. Eslam, J. Ampuero, M. Jover et al., "Predicting portal hypertension and variceal bleeding using non-invasive measurements of metabolic variables," Annals of Hepatology, vol. 12, no. 4, pp. 588-598, 2013. 


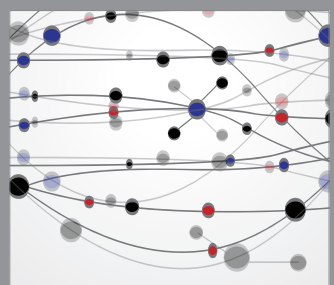

The Scientific World Journal
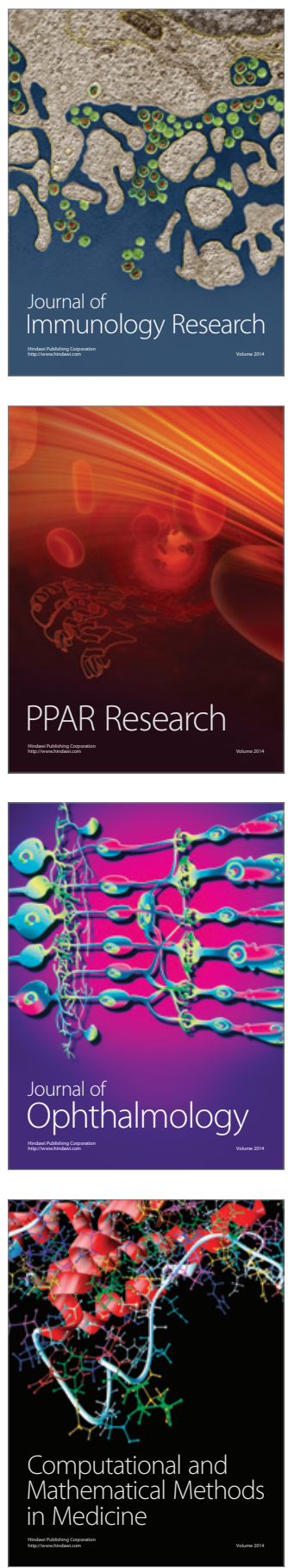

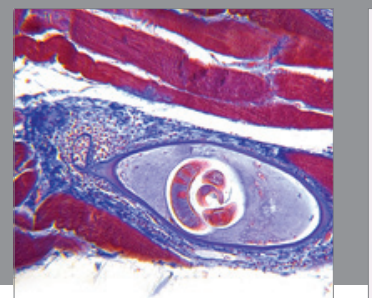

Gastroenterology

Research and Practice
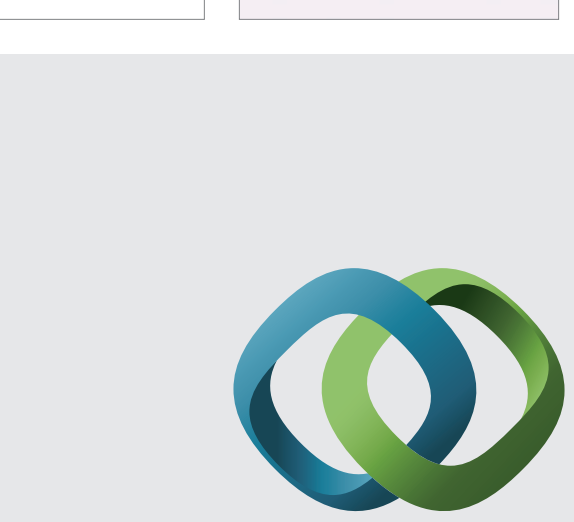

\section{Hindawi}

Submit your manuscripts at

http://www.hindawi.com
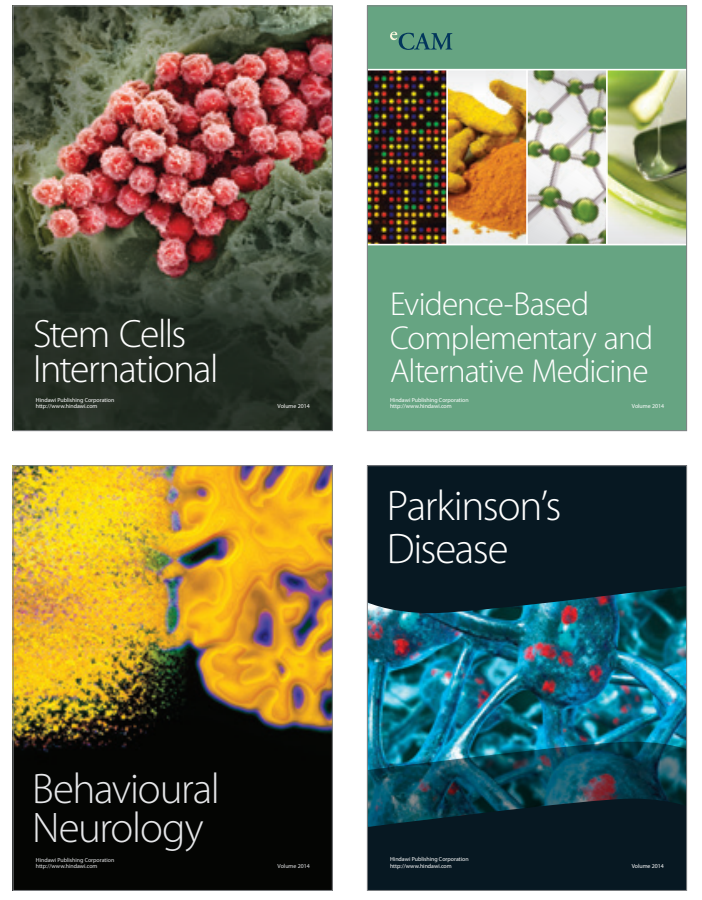
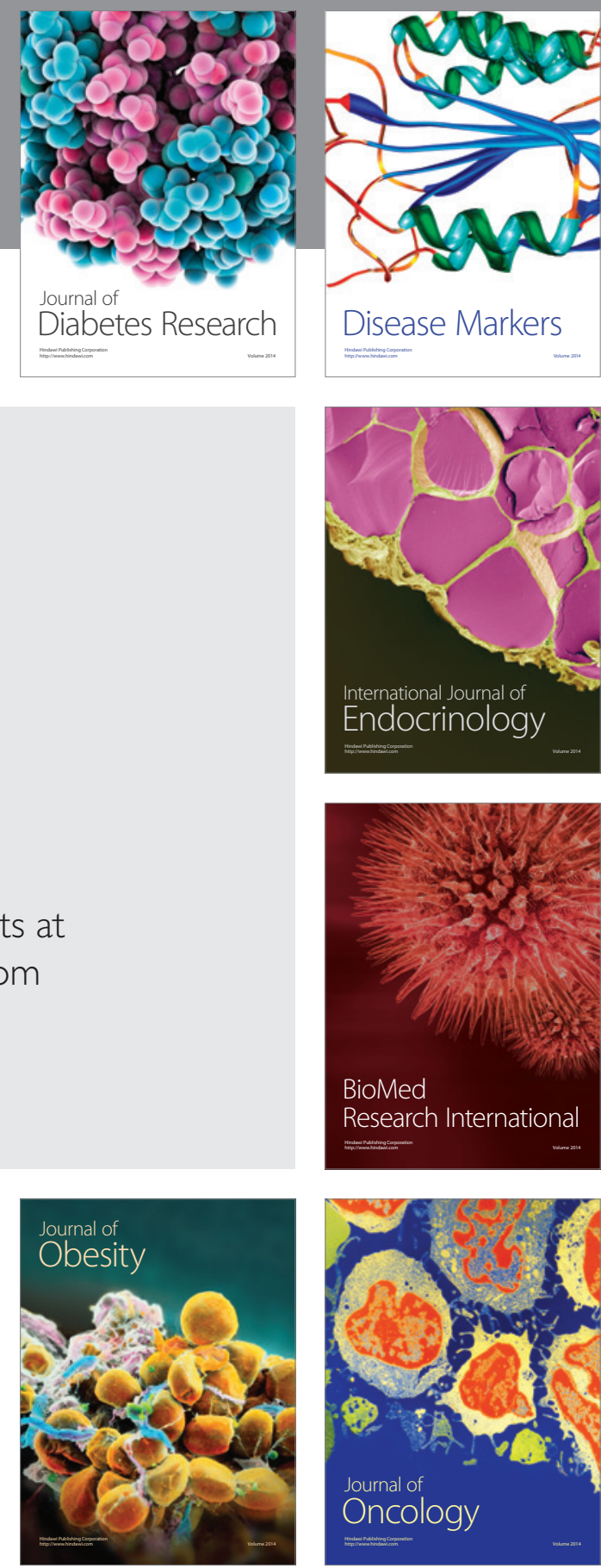

Disease Markers
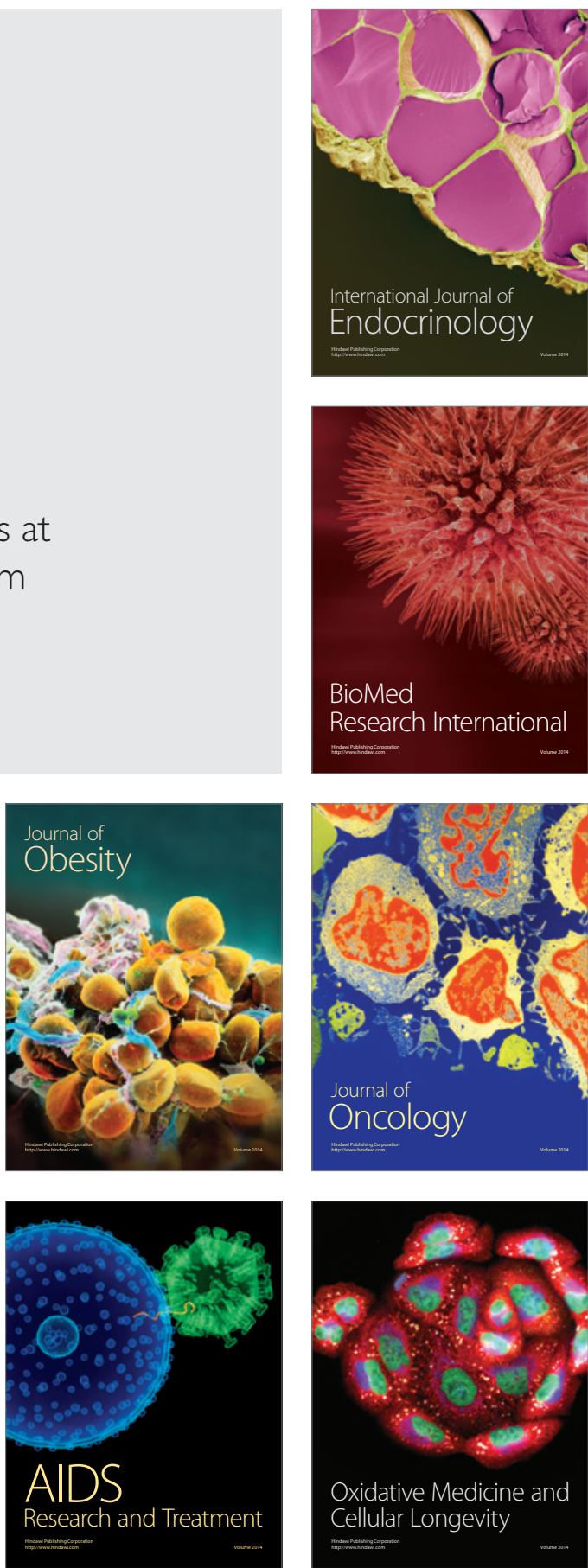